\title{
Atención de las personas que sufren trastornos adaptativos. Herramientas asistenciales para atención primaria y especializada de salud mental
}

\section{Health care of people suffering from adjustment disorders. Tools for primary and specialized mental health care}

\author{
Manuel Jesús Martínez López \\ Hospital Virgen del Rocío, Sevilla, España
}

\begin{abstract}
Resumen: La elevada demanda de atención en salud mental en el Sistema Sanitario Público de Andalucía requiere idear e implementar propuestas asistenciales proporcionales a la gravedad de los trastornos, a la vez que ágiles y de calidad. Con dicha finalidad el autor ha desarrollado tres herramientas asistenciales para la atención de personas que sufren trastornos adaptativos tanto en atención primaria como especializada de salud mental. Se trata de una guía de autoayuda, de un tratamiento psicológico grupal de sesión única y de una guía asistencial para los médicos de familia de atención primaria. Este trabajo contextualiza el tema, presenta las herramientas referidas y concluye la necesidad de valorar la penetración del uso de las guías descritas y la efectividad de las propuestas efectuadas.

Palabras clave: Trastornos adaptativos, atención, herramientas asistenciales, atención primaria, atención especializada de salud mental.
\end{abstract}

\begin{abstract}
The high demand for mental health care in the public health system of Andalusia requires to devise and implement care proposals proportional to the severity of the disorders which are also agile and of quality. For this purpose, the author has developed three tools to care for people suffering from adjustment disorders in both primary and specialized mental health care. Such tools include a self-help guide, a single-session psychological group treatment, and a care guide intended for primary health care family doctors. This article contextualizes the topic, presents the tools, and concludes the need to assess the penetration of use of the guides described and the effectiveness of the proposals made.

Keywords: Adjustment disorders, health care, assistance tools, primary health care, specialized mental health care.
\end{abstract}

La correspondencia sobre este artículo debe enviarse al e-mail: manuelm162@gmail.com

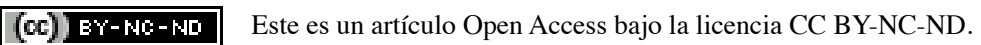


Un elevado número de personas sufren trastornos mentales en España. El proyecto ESMeD-España detectó que el 19,5\% de la población mayor de 18 años los sufrían en algún momento de su vida (Haro et al, 2006). En Atención Primaria (AP) más del $30 \%$ de las consultas tienen algún motivo psicológico, aunque Roca et al. (2009) han detectado uno o más trastornos mentales en más del 50\% de las personas atendidas en este nivel asistencial. Estos autores refieren que los trastornos más prevalentes eran los afectivos, seguidos de los de ansiedad y somatoformes. Respecto a los trastornos adaptativos (TA) Fernández et al. (2012) encontraron una prevalencia del 2’94 \% en centros de AP de Cataluña; y Labrador, Estupiñá y García-Vera (2010) señalan que el 7’36 \% de las personas atendidas en una clínica universitaria de Madrid en un período de nueve años presentaban este tipo de trastornos.

El III Plan Integral de Salud Mental de Andalucía 2016-2020 (Carmona et al., 2016) -PISMA III- informa que en el año 2006 la prevalencia de trastornos mentales en España entre personas de 15 a 65 años era del 13'8\%, siendo en Andalucía en 2013 del 14'8\%. En el año 2013, 114.641 pacientes fueron derivados desde los Centros de Salud (CS) de los distintos Distritos Sanitarios de Atención Primaria (DSAP) o desde las Áreas de Gestión Sanitaria hacia las Unidades de Salud Mental Comunitaria (USMC), donde se atendieron 268.521 personas; con un incremento del $25 \%$ en el total de usuarios en dichas unidades entre los años 2008 y 2013, y del $43 \%$ en el número total de consultas. Los trastornos neuróticos fueron los más atendidos, 65.768 , incluyéndose los TA. Estos datos revelan la alta necesidad de atención a la salud mental de la población andaluza y la elevada presión asistencial que reciben los dispositivos sanitarios de AP y Atención Especializada (AE).

Los TA son estados de malestar subjetivo acompañados de alteraciones emocionales que, por lo general, interfieren con la actividad social y que aparecen en el período de adaptación a un cambio biográfico significativo o a un acontecimiento vital estresante (Organización Mundial de la Salud [OMS], 1992). En su clasificación (CIE-10), la OMS diferencia 7 formas: Reacción depresiva breve, reacción depresiva prolongada, reacción mixta ansiedad-depresión, con predominio de alteración de otras emociones, con predominio de alteraciones disociales, con alteración mixta de emociones y disociales, otro trastorno de adaptación con síntomas predominantes especificados. En las dos primeras especifica un criterio temporal para el diagnóstico, y para la cuarta da ejemplos de síntomas posibles.

Existe controversia sobre el modelo de entendimiento de estos trastornos. Así Pérez-Sales (2009) distingue entre el que valida la prestación asistencial del sistema sanitario, al reconocer como patológicas las dificultades de adaptación al medio; y el que considera que existe una sobreprotección social y asistencial ante las inevitables emociones negativas en el ejercicio del vivir.

Azocar y Greenwood (2007) sostienen que, en general, los tratamientos psicológicos son considerados por muchos autores como los de elección para el abordaje de estos trastornos.

Vallejo (2015) pormenoriza distintos enfoques y técnicas cognitivo-conductuales usadas, como el entrenamiento en solución de problemas, entrenamiento en técnicas de relajación, intervenciones basadas en mindfulness; psicoterapia interpersonal, terapias grupales de apoyo, terapia familiar y técnicas de desensibilización y reproceso por el movimiento de ojos (Eye Movement Desensitization and Reprocessing, EMDR).

Recientemente se está promoviendo el uso de las tecnologías de la información y comunicación (programas computarizados, internet y dispositivos móviles) para el tratamiento psicológico (Simón, Molés y Quero, 2017). Así, Andreu-Mateu, Botella, Quero, Guillén y Baños (2012) y Quero et al. (2017), entre otros, están desarrollando experiencias de tratamiento de los TA mediante el uso de la realidad virtual.

Por otra parte se están implementando actualmente tratamientos psicológicos basados en el enfoque de transdiagnóstico. Tal y como señalan Sandín, Chorot y Valiente (2012), en dicho enfoque se entienden los trastornos mentales sobre la base de procesos cognitivos y conductuales causales y/o mantenedores de la mayor parte de aquéllos o de grupos consistentes de los mismos. Diversos autores han desarrollado protocolos de tratamientos psicológico para el abordaje de los trastornos mentales sustentados en ese enfoque (Barlow, Allen y Choate, 2004; Norton, 2012). Como los TA son - como los trastornos depresivos y de ansiedad - emocionales, también son objeto de este tipo de intervención. Dichos tratamientos están basados en los principios y técnicas de la terapia cognitivo-conductual, constan de módulos específicos, de un número de sesiones determinado y presentan variantes de aplicación individual o grupal y breve. Barlow et al. (2015) disponen también el uso de 
un manual para el paciente. Estos tratamientos se han demostrado efectivos en las variantes referidas (Newby, McKinnon, Kuyken, Gilbody, y Dalgleish, 2015).

En nuestro medio diversos autores están desarrollando intervenciones basadas en este enfoque. Así, Cano-Vindel (2011) ha diseñado un manual para el tratamiento grupal de trastornos emocionales en AP. Se compone de 7 sesiones de una hora y media de duración, desarrolladas a lo largo de 4 meses. El grupo está constituído por 8 a 12 personas. Este tratamiento se enmarca en el proyecto PsicAP (Psicología en Atención Primaria), por el que se trata de demostrar su eficacia en AP frente al tratamiento habitual. Los resultados preliminares así lo confirman (Infocop, 2017). Por su parte, Estévez, Ramos y Salguero (2017) también han hecho una propuesta de tratamiento para los trastornos emocionales basada en el Modelo General de los Desórdenes Emocionales.

Aunque los TA son objeto, en general, de terapias breves (Moles, 2016), no se han encontrado estudios específicos para su tratamiento psicológico en grupo en sesión única. Sin embargo, esta sesión única se usa desde hace varias décadas en los ámbitos del consejo psicológico y la psicoterapia, informándose de buenos resultados con problemas clínicos y relacionales (Talmon, 1990; Bloom, 2001; Hymmenm, Stalker y Cait, 2013). No obstante, el rigor metodológico de los estudios que avalan estos resultados ha sido objeto de crítica por Hymmenm, Stalker y Cait (2013).

\section{Proceso Asistencial Integrado Ansiedad-Depresión-Somatizaciones y materiales de autoayuda}

En la Comunidad Autónoma Andaluza, el Proceso Asistencial Integrado Ansiedad-Depresión-Somatizaciones (PAI-ADS) (Díaz del Peral et al., 2011) establece que los cuadros leves y moderados de ansiedad y depresión sean atendidos en primera instancia en AP. Entre otros diagnósticos se incluyen en este Proceso el de los TA y se señala como primera opción terapéutica para las personas incluidas en el mismo la psicológica y psicosocial. Así mismo, indica el uso de guías de autoayuda basadas en los principios de la terapia cognitivo-conductual como instrumento fundamental de trabajo en la intervención con la persona, que, en todo caso, se reduce a un número limitado de sesiones individuales presenciales o telefónicas, de corta o muy corta duración; y a un número de sesiones grupales también limitado. Este Proceso incorpora, así, las propuestas de las guías del National Istitute for Health and Clinica Excellence (NICE) $(2009,2011)$ sobre tratamiento de la depresión y la ansiedad, orientando el modelo de tratamiento hacia la práctica asistencial que en los últimos años se desarrolla en el Reino Unido (programa IATP: Improving Acces to Psychological Therapies). Sorprendentemente este Proceso no indica la incorporación de psicólogos en AP, a pesar de que prioriza las intervenciones psicológicas y psicosociales. Por otra parte, se define el espacio de colaboración como el elemento clave del PAI-ADS, en el que participan profesionales de AP y de Atención Especializada de Salud Mental (AE-SM) y en el que se articulan consultas conjuntas en los Centros de Salud, reuniones para análisis de casos y seguimiento del proceso y actividad docente.

En Andalucía la Consejería de Salud ha editado una Guía de autoayuda para la depresión y los trastornos de ansiedad (García-Herrera y Nogueras, 2011), elaborada por profesionales de la Unidad de Gestión Clínica de Salud Mental (UGC-SM) del Hospital Regional Universitario de Málaga y de la UGC Victoria del Distrito Sanitario Málaga. Esta guía consta de folletos a disposición de las personas que puedan beneficiarse de ellos, catorce relativos a la ansiedad y diecisiete a la depresión. A su vez, estos folletos conforman dos grupos: Unos de información y consejo, para ayudar a comprender lo que le está ocurriendo, y proporcionando unas primeras recomendaciones sobre cómo afrontar los síntomas; otros de actividades, centradas ya en estrategias concretas para facilitar la recuperación.

Los folletos de autoayuda para la ansiedad son los siguientes: Comprendiendo la ansiedad, Afrontando el estrés, ¿En qué consiste el trastorno de pánico?, ¿Qué es el trastorno de ansiedad generalizada?, Consejos para dormir mejor, ¿Qué son los tranquilizantes?, ¿Qué son los fármacos antidepresivos?, Aprenda a controlar sus pensamientos ansiosos, ¿Cómo hacer frente a las preocupaciones?, ¿Cómo resolver problemas?, Aprenda a organizar sus actividades, Aprenda a controlar su irritabilidad, Aprenda a relajarse, ¿Qué puedo hacer para dormir mejor? 
Y los de la depresión estos otros: ¿En qué consiste la depresión?, Afrontar la depresión posparto, ¿Qué puedo hacer para ayudarme a mí mismo si tengo depresión?, ¿Cómo puedo ayudar a un amigo o familiar con depresión?, ¿Cómo afrontar el duelo?, ¿Cómo mejorar mi ánimo con la actividad física?, Consejos para dormir mejor, ¿Qué son los fármacos antidepresivos?, ¿Qué son los tranquilizantes?, Mejore su autoestima, Aprenda a controlar sus pensamientos negativos, Cómo hacer frente a las preocupaciones, Aprenda a relacionarse mejor con los demás, ¿Cómo resolver problemas?, Aprenda a controlar la irritabilidad, Aprenda a organizar sus actividades, Mejore su estado de ánimo con la actividad física.

Por otra parte, la propia Consejería de Salud ha editado también Materiales para el afrontamiento saludable de las dificultades de la vida cotidiana (Servicio Andaluz de Salud, 2012). Los autores - entre otros, profesionales de la UGC-SM del Hospital Regional Universitario Carlos Haya, de Málaga - señalan que Ortiz y De la Mata (2004) han destacado que el personal sanitario atiende un incesante incremento de demandas que no se correlacionan con trastornos clásicos definidos en los manuales, de manera que se está produciendo una transformación de las demandas de la población y la respuesta técnica sanitaria que se está proporcionando es de dudosa eficacia.

Los propios autores también refieren que se ha subrayado que estas nuevas demandas tienen que ver con sentimientos de infelicidad o malestar estrechamente relacionados con los avatares de la vida cotidiana; relacionándose otras veces con sentimientos desagradables (tristeza, frustración, ira, odio, impotencia...) ante un acontecimiento o situación vital estresante o injusta, como respuesta adaptativa y no necesariamente patológica. Se trata de sufrimientos, rechazos o temores del entorno de cada paciente (De la Mata y Ortiz, 2007).

Los materiales incluyen las siguientes guías de autoayuda: Resolución de problemas, Afrontando las preocupaciones, Aprender a programar actividades placenteras, Aprender a relajarse, Autoestima, Concédete una oportunidad y cuídate; así como una Guía para profesionales. Como se observa, algunos de sus títulos son similares a los folletos referidos anteriormente.

Aunque en el conjunto de esta guías y materiales no se habla específicamente de los TA, tanto por los síntomas a que se refieren como por las áreas que se abordan y las propuestas de manejo, son herramientas aplicables para el abordaje de dichos trastornos.

La parte dedicada a la ansiedad de la mencionada Guía de autoayuda para la depresión y los trastornos de ansiedad ha sido incluída como material de trabajo en un programa de intervención denominado Grupo para el afrontamiento de la ansiedad en Atención Primaria (Quemada, Vargas, García-Herrera y Huizing, 2016), desarrollado por profesionales de tres UGC-SM de Málaga, del DSAP de Málaga y del Programa de Salud Mental del Servicio Andaluz de Salud. Dicho programa se implementa en AP, va dirigido a personas que sufren trastornos de ansiedad leves y moderados, tiene un enfoque psicoeducativo y se desarrolla a lo largo de 8 sesiones con frecuencia semanal de 2 horas de duración.

\section{Unidad de Salud Mental Comunitaria Oriente. Descripción y situación respecto a los trastornos adaptativos}

En la USMC-Oriente de la UGC-SM del Hospital Virgen del Rocío de Sevilla se atienden las solicitudes de AE-SM de 6 CS. El número total de tarjetas sanitarias que atienden es 105.000. El equipo de profesionales de la USMC-Oriente es multidisciplinar, estando integrado por tres psicólogos clínicos, cuatro psiquiatras, dos enfermeras, una trabajadora social, tres auxiliares de enfermería y una auxiliar administrativa. En 2015 se programaron 2.340 primeras consultas en esta Unidad. Los pacientes son derivados desde AP - la mayor parte - o AE y las consultas de urgencias hospitalarias. En la Unidad se atienden todo tipo de patologías mentales y de todas las franjas de edad. Tal y como recoge el PAI-ADS existen espacios de colaboración con todos los CS cuyas solicitudes se atienden.

Respecto a los TA constatamos en dichos espacios y en las derivaciones de casos a la Unidad que, frecuentemente, las personas buscan ayuda en sus médicos de familia para aliviar el sufrimiento emocional que producen los golpes de la vida; $y$, aunque éstos las atienden con éxito en muchos casos con los mejores procedimientos de que disponen, existen diversos factores que complican su abordaje en AP: el elevado número de 
personas a las que atender, la escasísima disponibilidad de tiempo para atender a cada paciente, su intervención a distintos niveles en la práctica totalidad de los PAI, la falta de formación en un área - la psicológica - que no es propiamente de su especialidad (y tampoco su competencia directa), las peticiones de los pacientes de recibir atención especializada y la ausencia de profesionales de la psicología en este nivel. Todo esto implica que se deriven a AE-SM - incluso, a veces, con carácter urgente - a personas que sufren TA; o, también, a personas que sufren emocionalmente por un acontecimiento vital difícil acontecido muy recientemente y de dudoso diagnóstico TA. Muchos de estos casos llegan con tratamiento farmacológico ya indicado (en contra de lo que indica el mencionado proceso).

En definitiva, llegan a SM un elevado número de pacientes que tienen un sufrimiento inevitable que se produce por el hecho de vivir, que se patologiza por la cultura del estado del bienestar y que, según el PAI-ADS, no deberían de ser atendidos en AE sino en AP, donde, como hemos visto antes, existen limitaciones de medios y de profesionales que permitan hacerlo plenamente.

Por tanto, la elevada demanda de atención en nuestra Unidad, la diversidad de patologías que se atienden y los distintos grados de gravedad de las mismas, han aconsejado desarrollar propuestas asistenciales proporcionales y ágiles; y que, específicamente, permitan atender a las personas que sufren TA con la mejor calidad posible (para que no se cronifique el malestar) sin sobreatender (para que no se patologice la normalidad).

\section{Herramientas asistenciales para atención primaria y especializada de salud mental}

El autor ha desarrollado en la USMC-Oriente tres iniciativas para el abordaje de los TA: la elaboración y difusión de una guía de autoayuda - Pérdidas en la vida: Una guía de autoayuda para aliviar el sufrimiento que producen -, la elaboración e implementación de un programa de tratamiento psicológico grupal de sesión única (Martínez, 2017) y la elaboración y difusión de una guía para médicos de familia para la atención de personas que sufren trastornos adaptativos - Guía asistencial con personas que sufren trastornos adaptativos en Atención Primaria-.

Las dos primeras se refieren a intervenciones con las personas que sufren TA y la tercera es una propuesta organizativa de la atención a las mismas en AP, en que se sugieren los pasos a seguir en la oferta asistencial.

Partiendo de la conceptualización de los estresores vitales como pérdidas del status quo del bienestar que se tiene en cada momento en diferentes situaciones y áreas de la vida, tanto con el tratamiento psicológico grupal como con la guía de autoayuda mencionados se persigue ayudar a la persona a posicionarse ante dichas pérdidas de una forma diferente para aliviar su sufrimiento. Uno y otra incluyen y adaptan método y contenidos que son objeto del tratamiento psicológico individual del autor con las personas a las que atiende en la USMC-Oriente con diagnóstico de TA. La mayor parte de las veces son tratamientos de sesión única tras la cual se da el alta a la persona. La sesión incluye la recepción de la demanda, el conocimiento las circunstancias de vida de la persona y de sus mecanismos de funcionamiento, la identificación de las fuentes de la pérdida de bienestar que está sufriendo, la identificación de áreas de su vida satisfactorias y fuentes de bienestar, la vida como un proceso de cambio, la comprensión de su malestar emocional en el conjunto de todo lo anterior, la propuesta de un nuevo enfoque y afrontamiento de su situación, tareas para casa, medidas de aliento y cierre de la sesión.

\section{Guía de autoyuda}

La guía Pérdidas en la vida: Una guía de autoayuda para aliviar el sufrimiento que producen (Anexo 1) se elaboró con el propósito de disponer de un instrumento de ayuda para la prolongación del tratamiento con personas que sufren TA más allá de la sesión en la Unidad. Una vez elaborada se presentó y entregó a los profesionales de la misma por si veían en ella también una ayuda para su trabajo con personas con estos trastornos. Y se propuso su presentación en cada espacio de colaboración de los CS para ponerla a disposición de los médicos en su abordaje de dichos trastornos, cosa que finalmente hizo el autor en cada uno de los centros. 
La guía tiene un estilo narrativo, conformando un relato en que destacan los siguientes apartados de contenidos: la inevitable realidad de los cambios en la vida, las pérdidas y ganancias como expresión de dichos cambios, el sufrimiento emocional producido por las pérdidas como una herida que precisa cuidados para curar y los cuidados que podemos proporcionarnos las personas para recuperarnos emocionalmente de las pérdidas.

\section{Tratamiento psicológico grupal de sesión única}

La metodología del tratamiento permite su aplicación a grupos numerosos, en sesión única de cuatro horas de duración. Se desarrolla a través de 4 fases.

Fase 1. Las pérdidas en la vida y el sufrimiento emocional como expresión de que estamos vivos. Herida-sufrimiento-cicatriz-huella. Las heridas necesitan cuidados y tiempo para cicatrizar. Vivir con las huellas de la pérdida.

Fase 2. Lo que la psicología sabe de lo que no ayuda a las personas a recuperarse emocionalmente de las pérdidas sufridas: huir del sufrimiento, lamentarse y recrearse en él, decirse que uno no se recuperará jamás de esto, responsabilizar a otros de la posibilidad de recuperarse, aislarse, descuidar lo que uno sigue teniendo, rechazar cualquier buena sensación que uno tiene, rechazar posibilidades que llamen a la puerta de la vida.

Fase 3. Lo que la psicología sabe de lo que sí ayuda a las personas a recuperarse emocionalmente de las pérdidas: sentir el sufrimiento, agradecer lo que le ha dado a uno aquello que ha perdido hasta que se produjo la pérdida, centrarse en lo que depende de uno hacer para sentirse mejor, mantener el contacto con el mundo, tener muy presente lo que uno sí sigue teniendo y cuidarlo, estar atento a las buenas sensaciones que uno tiene.

Fase 4. El bienestar se conquista con las consideraciones y las acciones que uno desarrolla en su vida diaria. Para lo que nos ocupa, los medicamentos psicológicos requieren más esfuerzo del paciente que los medicamentos químicos: el paciente tiene que aplicar diariamente en su vida lo que se sabe que le ayudará a recuperarse emocionalmente.

Cada una de estas fases se desarrolla a través de las 3 siguientes subfases:

Introducción por el psicólogo clínico de las ideas centrales y contenidos de la fase correspondiente.

Trabajo en pequeños grupos (3-4 personas) respondiendo cada uno oralmente a las preguntas escritas que se formulan (anexo 2) y que versan sobre las ideas centrales y contenidos de dicha fase; en sus respuestas se dirigen a las otras personas del pequeño grupo.

Puesta en común e intervención del profesional que aplica el tratamiento para transmitir un modelo saludable de funcionamiento.

La descripción del programa, una contextualización del tema - que se incorpora en la introducción de este trabajo - y una evaluación preliminar del mismo ha sido presentada por el autor (Martínez, 2017).

\section{Guía asistencial para Atención Primaria}

Por otra parte, con el propósito de guiar a los médicos de familia en la atención de las personas que sufren TA se elaboró la referida Guía asistencial con personas que sufren trastornos adaptativos en Atención Primaria (Anexo 3). Ésta consta de 4 apartados: diagnóstico, curso y pronóstico de los trastornos adaptativos; uso de la guía de autoayuda; indicación de tratamiento psicológico en grupo de una sola sesión; derivación ordinaria a la USMC-Oriente. A través de dichos apartados se dan orientaciones básicas para que los médicos puedan situarse respecto a este trastorno y complementar y secuenciar la atención que ya dan a las personas que lo sufren con dos opciones de intervención más antes de su derivación convencional — valoración individual en primera consulta - a la USMC, como son la guía de autoayuda y el tratamiento psicológico grupal de sesión única. 


\section{Conclusión}

Teniendo en cuenta la alta demanda de atención por problemas de salud mental en la sanidad pública se tienen que desarrollar propuestas asistenciales proporcionales, ágiles y de calidad. Con este propósito en la UGC-SM del Hospital Virgen del Rocío (Sevilla) el autor de este trabajo ha ideado y desarrollado tres herramientas para el abordaje de personas que sufren TA, que son las que se han presentado aquí. Por sus características y la población a la que se dirigen, dichas herramientas son aplicables a otros contextos diferentes a aquél en el que surgen. Como se ha señalado anteriormente, una de ellas, la del tratamiento psicológico grupal de sesión única, está en fase de evaluación, habiéndose presentado datos preliminares al respecto. No así en el caso de la otras dos. Aunque las Guías NICE y el Servicio Andaluz de Salud promueven e indican el uso de guías de autoayuda para trastornos del PAI-ADS, es esencial conocer la penetración de su uso entre los profesionales de AP y AE de SM y la consideración de los propios pacientes de su utilidad y de éstos y los profesionales de su influencia en la recuperación. Esta valoración debe realizarse también respecto a la guía de manejo asistencial para los médicos de AP, en los propios espacios de colaboración con AP e individualmente. Por tanto, nuevos estudios al respecto de todo ello deben ser realizados.

\section{Referencias}

Andreu-Mateu, M. S., Botella, C., Quero, S., Guillén, V. y Baños, R. M. (2012). La utilización de la realidad virtual y estrategias de psicología positiva en el tratamiento de los trastornos adaptativos. Behavioral Psychology/Psicología Conductual, 20, 323-348.

Azocar, F. y Greenwood, G. (2007). Service use for patients with adjustment disorder and short term treatment: A brief report. The Internet Journal of Mental Health, 4(2). Recuperado de https://print.ispub.com/api/0/ ispub-article/6083

Barlow, D. H., Allen, L. B. y Choate, M. L. (2004). Toward a unified treatment for emotional disorders. Behavior Therapy, 35, 205-230.

Barlow, D., Farchione, T., Fairholme, C., Ellard, K., Boisseau, C., Allen, L. y Ehrenrich-May, J. (2015). Protocolo unificado para el tratamiento transdiagnóstico de los trastornos emocionales. Madrid, España: Alianza Editorial.

Bloom, B. (2001). Focused single session psychotherapy: A review of the clinical and research literature. Brief Treatment and Crisis Intervention, 1(1), 75-86.

Carmona, J. (Dir.), García-Cubillana, P., Millán, A., Huizing, E., Fernández-Regidor, G., Rojo, M. y Aguilera, L. (2016). III Plan Integral de Salud Mental de Andalucía: 2016-2020. Sevilla, España: Consejería de Salud. Recuperado de http://www.juntadeandalucia.es/servicioandaluzdesalud/principal/documentosacc.asp?pagina=pr_GestCal_SM_3

Cano-Vindel, A. (2011). Bases teóricas y apoyo empírico de la intervención psicológica sobre los desórdenes emocionales en atención primaria. Una actualización Ansiedad y Estrés, 17, 157-184.

De la Mata I., y Ortiz, A. (2007). La colonización psiquiátrica de la vida. Archipiélago. Cuadernos de crítica de la cultura, 76, 39-50.

Díaz del Peral, D. (Coord.), Aragón, J., Aranda, J., Bellón, J., Bordallo, A., Cantero, L., ... Sanz, R. (2011). Ansiedad, depresión, somatizaciones: Proceso Asistencial Integrado. Sevilla, España: Consejería de Salud. Recuperado de http://www.juntadeandalucia.es/salud/export/sites/csalud/galerias/documentos/p_3_p_3_procesos_asistenciales_integrados/ansiedad_depresion_somatizaciones/ansiedad_depresion_somatizacion_2e.pdf

Estevez, A., Ramos, J. y Salguero, J. M. (2015). Tratamiento transdiagnóstico de los desórdenes emocionales. Madrid, España: Síntesis.

Fernández, A., Mendive, J. M., Salvador-Carulla, L., Rubio-Valera, M., Luciano, J. V., Pinto-Meza, A., y Serrano-Blanco, A. (2012). Adjustment disorders in primary care: Prevalence, recognition and use of services. British Journal of Psychiatry, 201(2), 137-142. https://doi.org/10.1192/bjp.bp.111.096305

García-Herrera, J. M. y Nogueras, E. V. (Coords.) (2011). Guía de autoyuda para la depresión y los trastornos 
de ansiedad. Sevilla: Consejería de Salud. Recuperado de http://www.juntadeandalucia.es/servicioandaluzdesalud/principal/documentosacc.asp?pagina=gr_smental_23_12_gauto

Haro, J., Palacín, C., Vilagut, G., Martínez, M., Bernal, M., Luqye, I., ... Alonso, J. (2006). Prevalencia de los trastornos mentales y factores asociados: resultados del estudio ESMeD-España. Medicina Clínica, 126, 445-451.

Hymmen, P., Stalker, C. y Cait, C.A. (2013) The case for single-session therapy: Does the empirical evidence support the increased prevalence of this service delivery model? Journal Mental Health, 22(1), 60-71. https://doi.org/10.3109/09638237.2012.670880

Infocop online (28 de febrero de 2017). Los pacientes que reciben intervención psicológica en AP se recuperan de sus síntomas cuatro veces más que los que reciben el tratamiento habitual. Recuperado de http://www. infocop.es/view_article.asp?id=6687

Labrador, F. J., Estupiñá, F. J. y García Vera, M. P. (2010). Demanda de atención psicológica en la práctica clínica: tratamientos y resultados. Psicothema, 22, 619-626. Recuperado de http://www.psicothema.com/ psicothema. .asp? id=3776

Martínez, M. J. (2017). Tratamiento psicológico grupal de sesión única para personas que sufren trastornos adaptativos. Programa y evaluación preliminar. Apuntes de Psicología ,35(2), 131-140. Recuperado de http:// www.apuntesdepsicologia.es/index.php/revista/article/view/666/486

Molés, A. (2016). Eficacia diferencial de dos formas de aplicación de las tareas para casa en el tratamiento de los Trastornos Adaptativos: aplicación apoyada por las TICs versus aplicación de forma tradicional (Tesis doctoral, Universitat Jaume I, España). Recuperado de http://www.tdx .cat/bitstream/handle/10803/378368/ mmoles.pdf?sequence $=1$

National Institute for Health and Clinical Excellence (2009). Depression in adults with a chronic physical health problem: Treatment and management. NICE Clinical Guideline, 91. Recuperado de http://www.nice. org.uk/CG91niceguideline

National Institute for Health and Clinical Excellence (2011). Generalised anxiety disorder and panic disorder (with or without agoraphobia) in adults: Management in primary, secondary and community care. NICE Clinical Guideline, 113. Recuperado de http://guidance.nice.org.uk/CG113/NICEGuidance/pdf/English

Newby, J. M., McKinnon, A., Kuyken, W., Gilbody, S. y Dalgleish, T. (2015). Systematic review and metaanalysis of transdiagnostic psychological treatments for anxiety and depressive disorders in adulthood. Clinical Psychological Review, 40, pp.91-110. https://oi.org/10.1016/j.cpr.2015.06.002

Norton, P. J. (2012). Group cognitive-behavioral therapy of anxiety: A transdiagnostic treatment manual. Nueva York, Estados Unidos: Guilford.

Organización Mundial de la Salud (OMS) (1992). CIE 10 - Trastornos Mentales y del Comportamiento. Madrid, España: Meditor.

Ortiz A. y De la Mata, I. (2004). Ya es primavera en Salud mental. Sobre la demanda en tiempos de mercado. Átopos, 2(1), 15-20.

Pérez-Sales, P. (2009). Trastornos adaptativos y reacciones de estrés. En T. Palomo y M. A. Jiménez- Arriero (Eds.), Manual de Psiquiatría (pp. 403-417). Madrid, España: Asociación Española de Neuropsiquiatría.

Quemada González, C., Vargas Salazar, J., García-Herrera, J. M. y Huizing, E. (Coords.) (2016). Grupo de afrontamiento de la ansiedad en Atención Primaria. Sevilla, España: Servicio Andaluz de Salud. Recuperado de http:// www.juntadeandalucia.es/servicioandaluzdesalud/publicaciones/Listadodeterminado.asp?idp=653

Quero, S., Andreu-Mateu, S., Moragrega, I., Baños, R.M., Molés, M., Nebot, S. y Botella, C. (2017). Un programa cognitivo-conductual que utiliza la realidad virtual para el tratamiento de los trastornos adaptativos: una serie de casos. Revista Argentina de Clínica Psicológica, XXVI, 5-18. Recuperado de http://4www. redalyc.org/articulo.oa?id=281950399002

Roca, M., Gili, M., García-García, M., Salva, J., Vives, M., García Campayo, J. y Comas, A. (2009). Prevalence and comorbility of common mental disorders in primare care. Journal of affective disoders, 119(1-3), 52-58. https://doi.org/10.1016/j.jad.2009.03.014

Sandín, B., Chorot, P. y Valiente, R. M. (2012). Transdiagnóstico: Nueva frontera en psicología clínica. Revista de Psicopatología y Psicología Clínica, 17(3), 185-203. 
Servicio Andaluz de Salud (2012). Materiales para el afrontamientos saludable de las dificultades de la vida cotidiana. Granada, España: Autor. Recuperado de http://www.juntadeandalucia.es/servicioandaluzdesalud/ publicaciones/Listadodeterminado.asp?idp $=481$

Simón, N., Molés, M. y Quero, S. (2017). Tratamientos psicológicos para los trastornos adaptativos. Àgora de salut, IV, 377-386. https://doi.org/10.6035/AgoraSalut.2017.4.39

Talmon, M. (1990). Single-session therapy: Maximizing the effect of the first (and often only) therapeutic encounter. San Francisco, Estados Unidos: Jossey-Bass.

Vallejo, B. (2015). Personalidad, afrontamiento y positividad en pacientes con trastorno adaptativo (Tesis doctoral), UNED, España. Recuperado de http://e-spacio.uned.es/fez/eserv/tesisuned:Psicologia-Bvallejo/ VALLEJO_SANCHEZ_Beatriz.pdf

Artículo recibido: 23/02/2018

Artículo aceptado: 04/06/2018 


\section{ANEXO 1}

\section{Pérdidas en la vida: Una guía de autoayuda para aliviar el sufrimiento que producen}

Usted ha acudido a su médica/o de familia buscando ayuda porque se encuentra mal emocionalmente: deprimida/o, angustiada/o, irritada/o, insomne, inapetente, ...

Esto puede que le esté ocurriendo desde que ha sufrido un cambio en su vida, perdiendo algo que usted lo consideraba importante para su bienestar.

La vida está llena de cambios, desde el nacimiento hasta la muerte, que es el cambio definitivo.

Cuando se nace se empieza un largo camino en el que se estará continuamente perdiendo y ganando cosas. El recién nacido pierde el contacto íntimo con su madre en el útero de ésta. Deja de estar cálidamente protegido y sale a un nuevo mundo que le espera. Le toman en brazos, le sonríen, le dan de mamar, le acarician, ... Pierde algo que apreciaba mucho y gana cosas que empieza a apreciar y que deseará no perder. Sin embargo, esas nuevas cosas cambian, se pierden, y llegan otras diferentes. Cada cambio genera sufrimiento en menor o mayor grado: la persona se entristece porque ya no le acompaña algo que le gratificaba y se angustia porque reconoce que puede perder cosas de las que ya tiene y porque teme no conseguir otras nuevas que sean una fuente de bienestar como lo era aquella que se fue.

¿Qué es lo que se puede perder en la vida? Todo. Enferman y fallecen familiares, amigos, conocidos; enferma una/o mismo; se cambia de vivienda, de barrio, de colegio, de centro de trabajo; se van los amigos; se pierde el empleo, se reducen los ingresos económicos; se complican las relaciones con familiares, amigos, compañeras/ os de trabajo; se rompe la pareja, se independizan hijos/as, ... Se llega a perder hasta la propia vida.

Las pérdidas en la vida son como golpes o cortes que nos producen hematomas, fracturas, heridas, lesiones en definitiva, en este caso en forma de sufrimientos emocionales. Todas las lesiones causan dolor en menor o mayor grado y requieren tiempo y cuidados para que mejoren y curen. Si no se cuidan, las heridas se pueden infectar, complicarse, hacer enfermar otras partes del cuerpo que estaban sanas y dejar cicatrices feas. También ocurre esto con las heridas emocionales que producen las pérdidas. Hay que cuidarlas para que cicatricen debidamente y tratar de que las huellas que dejen sean las menores posibles.

Por tanto, en este guía se describen cuidados que las personas se pueden proporcionar para recuperarse emocionalmente de las pérdidas de la vida. Le invitamos a que lea este documento una y otra vez, con interés, reflexionando sobre lo que aquí se dice, llevando a su vida diaria con constancia y paciencia las propuestas que se hacen; e, incluso, incorporando otras medidas que, aunque no se consideren aquí, usted crea que puedan ser buenas para recuperarse.

Sufrir - al igual que disfrutar - es inevitable por el hecho de estar vivos. Con las pérdidas las personas sufrimos a veces tanto que sentimos que no podemos soportarlo y que no nos podremos recuperar jamás del golpe sufrido. Sin embargo, la experiencia demuestra que la mayor parte de las personas nos recuperamos de las pérdidas en la vida, también de las más graves (muertes de seres queridos), a veces incluso sin ayuda de profesionales.

Profesionales y personas cercanas pueden ayudar a la recuperación pero ésta dependerá fundamentalmente de sí misma/o. Es a una/o misma/o a quien le corresponde autoaplicarse los cuidados que se precisan para que las heridas sanen. Y es una buena noticia que la propia recuperación dependa de una/o mismo y no de otras personas porque lo se puede controlar es lo que depende de sí misma/o.

Como es lógico, procuramos sufrir lo menos posible y para eso, a veces, se recurre a vías de escape que alejan artificialmente a la persona del sufrimiento abocándola a volvérselo a encontrar incluso en más alto grado. Es por eso que algunas personas se refugian en el consumo de alcohol y otras drogas o pretenden abusar de fármacos.

La psicología sabe, sin embargo, que la mejor manera de tratar el sufrimiento es acogerlo y aceptarlo, pero sin recrearse en él. No es fácil. Lo que se ha perdido se hace presente en la cabeza una y otra vez y el corazón sufre por la pérdida. No es que queramos pensarlo, es que los pensamientos nos asaltan, nos invaden, invitándonos a quedarnos pegados a ellos, reinando, cavilando, lamentándonos. Todo esto es un proceso natural que, probablemente, no sólo sirva para descargar el sufrimiento sino también para digerir la pérdida que se ha su- 
frido. Pero cuando la cavilación y la lamentación se convierten en el centro de la vida de la persona por mucho tiempo ésta deja de dirigir su energía y su atención hacia tareas que le puedan ayudar a seguir avanzando en su recuperación.

¿Cómo podemos conseguir des-pegarnos de los pensamientos que nos atormentan? Entre otras maneras, concentrándonos en la respiración (suave y por la nariz); ocupándonos en tareas manuales que requieran mucha atención; leyendo textos sencillos, cortos; paseando por espacios naturales (parques, por ejemplo) depositando la mirada en árboles y pájaros, y el oído en los sonidos de éstos. Y, desde luego, desarrollando los siguientes cuidados que a continuación también se van a seguir describiendo.

Nos embarga la pena cuando perdemos en la vida. A veces no encontramos consuelo en el recuerdo de seres queridos que se han ido, de salud que perdemos, de trabajos de los que nos despiden y de tantas otras situaciones de pérdidas. Pero, quizá, en algún momento surja el recuerdo amable y agradecido por lo que tuvimos aunque ya no lo tengamos. Este es otro de los cuidados que nos podemos dar: reconocer lo que se nos dio, apreciarlo y agradecer el tiempo que estuvo con nosotros.

En los malos momentos no se tienen ganas de nada, nos sentimos inquietos/as, tristes, incómodas/os. Se abandonan actividades que se desarrollaban e, incluso, se evita el contacto con otras personas. Se interrumpe el orden de vida habitual porque no nos sentimos bien. De esta forma, el vagón que somos se suelta del convoy y el tren de la vida avanza en su camino, quedándonos cada vez más descolgados de él. No es fácil seguir adelante cuando nos encontramos así. Sin embargo, ni el aislamiento ni la inactividad nos ayudarán a recuperarnos. Se sabe que las personas no nutrimos de las pequeñas y grandes situaciones cotidianas que dan contenido a la vida. Y si habiendo perdido alguna cosa importante para nosotros nos retiramos de otras que sí seguimos teniendo, lo que conseguimos es dejar de beneficiarnos de lo bueno que nos aportan y tener la sensación de que nuestra vida se va convirtiendo en un páramo estéril. Por eso, cuando se pierde algo importante lo que hay que hacer es cuidar con más esmero, si cabe, lo que sí seguimos teniendo: familia, amistades, aficiones, trabajo, pertenencias, el propio cuerpo, ... A veces ocurre que en la desesperación de nuestro sufrimiento descargamos nuestro malestar en quienes están más cerca de nosotros. Lo hacemos sin pensar, pero cuando nos damos cuenta de que estamos atacando a quienes apreciamos, nos sentimos culpables y el sufrimiento aumenta.

Nos acostumbramos a un mundo que nos da seguridad. Luchamos por alcanzar logros que entendemos que nos ayudarán a vivir bien. Nos agarramos a ellos y deseamos que permanezcan siempre ahí. Sin embargo, la vida no es estable. Nos da y nos quita cosas continuamente. Pero si nos encerramos en nosotros mismos cuando hemos perdido algo, cerramos la puerta a posibles nuevos aportes que la vida nos traiga, quizá llenándonos de nuevas satisfacciones. Nuevas amistades, vínculos familiares ya existentes que se renuevan o fortalecen, nuevos gustos, en fin nuevos intereses en la vida.

¿Es posible sentirse bien en algún momento si hemos sufrido una pérdida importante en la vida? (por ejemplo, en el caso de una separación, la muerte de un padre o una madre o la pérdida de un empleo). Inicialmente, bajo el impacto de la pérdida, es difícil que esto ocurra. Sin embargo, si uno no se resiste a vivir, reconoce la totalidad de ingredientes que conforman su vida (situaciones, actividades, personas) y los tiene presentes, llegará al momento en que se descubra tranquilo, animado, incluso disfrutando. Y no es que se sea inconsciente o una mala persona. Simplemente esto ocurre sólo por el mero hecho de estar vivo. Reconocer las buenas sensaciones, aceptarlas y cuidarlas es importante para avanzar en la propia recuperación.

¿Conviene hablar del sufrimiento que se tiene? Desde luego que sí. Hacerlo con personas cercanas de nuestro entorno que sean capaces de escuchar, de decir palabras de consuelo, ánimo y ofrecer buenos consejos en nuestro favor. Pero también conviene interesarse por los que están a nuestro lado, personas a las que apreciamos y que tiene una vida que de alguna manera comparten con nosotros en mayor o menor medida. Salir de nosotros para estar con los otros es una manera saludable de reconocer la amplitud de nuestro mundo.

Hay pérdidas que requieren mucha acción. Si uno pierde el trabajo no tendrá más remedio que, aunque esté triste y angustiado, ponerse inmediatamente a buscar uno nuevo. Se puede tener la tentación de no tener esperanza de encontrarlo pero, en ocasiones, esta desesperanza inmoviliza a la persona, dejando de hacer las gestiones necesarias para volver a tener la posibilidad de emplearse.

Ya se ha mencionado la concentración en la respiración suave y por la nariz como un buen método para que se desprendan los pensamientos que nos hacen sufrir. Vamos a decir algo más sobre esto. Las personas tenemos 
la capacidad de autocalmarnos aunque no la hayamos desarrollado. En los colegios se nos enseñan muchos conocimientos pero, salvo excepciones, no éste precisamente. Las técnicas de relajación sirven para eso, para aprender a autocalmarse. Existen diferentes tipos de técnicas constrastadamente útiles. Pero las técnicas de relajación sirven para calmarse cuando se está angustiada/o si la persona la ha practicado mucho antes.

Usted puede practicar esta sencilla técnica que, a continuación, se describe. Todas las noches, en la cama, antes de echarse a dormir, con los ojos cerrados, prestará atención a su respiración, que será suave y por la nariz. Sentirá el aire rozar los orificios de la nariz al entrar y salir. Y pasados unos minutos concentrada/o en la respiración, cada vez que expulse el aire por la nariz suavemente, al mismo tiempo aflojará el cuerpo como si fuera un globo que se desinfla; haciéndolo una y otra vez en cada expiración. Cuando se dé cuenta de que se ha distraído volverá a poner la atención en la respiración, suave y por la nariz. Lo hará durante un rato. Si se duerme no pasa nada. Pero no pretenda dormirse ni relajarse. El objetivo es sólo practicar la técnica.

Cuando lleve varias semanas practicando todas las noches y usted crea que conoce bien la técnica, seguirá practicándola por las noches, y también empezará a hacerlo durante el día, dos veces, en momentos en que usted no esté especialmente inquieto/a (por ejemplo, mientras que ve la televisión o cuando reposa después de almorzar). La técnica siempre es la misma. Así que durante varias semanas más practicará por las noches y también dos veces durante el día. De tal manera que cuando usted sepa que domina la técnica y que ésta le ayuda a autocalmarse será el momento en que la deberá usar en momentos de más ansiedad. Por tanto, seguirá practicando por las noches, lo seguirá haciendo durante el día dos veces y, por último, como se le está explicando ahora, la usará cuando está especialmente ansioso/a. Aunque estos momentos de más ansiedad serán probablemente menos intensos y frecuentes sólo por el mero hecho de hacer la práctica diaria de noche y dos veces al día.

Dos últimas recomendaciones para su autocuidado.

Está demostrado que el ejercicio físico es un buen calmante y un buen antidepresivo para las personas. Al menos salga a andar todos los días, un buen rato. Hágalo por donde usted guste. Los parques son recomendables pero hay personas que les gusta hacerlo por zonas más concurridas, comerciales incluso. Puede hacerlo sola o acompañado. Si se hace acompañado se puede ir charlando con la/s otra/s persona/s, distrayéndose con distintos contenidos de la conversación.

Por último, el Servicio Andaluz de Salud ha publicado unas Guías de Autoayuda para personas que sufren ansiedad o depresión. Las pueden consultar escribiendo en el buscador de internet "Guías de autoayuda del Servicio Andaluz de Salud" o copiando y pegando la siguiente dirección: http://www.juntadeandalucia.es/ servicioandaluzdesalud/principal/documentosacc.asp?pagina=gr_smental_23_12_gauto

Las Guías se pueden imprimir en la misma página web.

Hay diferentes guías que tratan de asuntos específicos de interés para entender y afrontar el malestar ansioso o depresivo que sufren las personas. Lea los títulos de todas ellas y entre en las que llamen su atención. Deberá leerlas detenidamente, reflexionar sobre los que se dice en cada una de ellas e interiorizar y llevar a la práctica las consideraciones y propuestas que se hagan.

Las maneras de autocuidarse no se acaban aquí. Íntimamente las personas sabemos, en general, lo que nos pueden venir bien para recuperarnos de los golpes de la vida. Dediquemos un tiempo diario no sólo a darnos cuenta de cómo nos sentimos, sino también a reflexionar sobre lo que creemos que nos puede ayudar a sentirnos mejor.

No olvide que conseguir bienestar cuando una/o se encuentra mal emocionalmente es el resultado de un camino en que la personas ponen las medidas que le indican los profesionales. No basta con hablar con ellos, es necesario hacer lo que se les dice. Con esta Guía de Autoayuda (y cualquier guía de autoayuda) ocurre lo mismo: no basta sólo con leerla una vez. Hay que hacerlo muchas veces y llevar a la práctica lo que se dice en ella. 


\section{ANEXO 2}

Preguntas a responder en el trabajo en pequeños grupos del tratamiento psicológico grupal de sesión única

Fase 1:

¿Cree que es posible vivir sin sufrimiento?

¿Se ha hecho alguna herida recientemente? ¿Le dolió? ¿Cuánto tiempo tardaron las molestias en desaparecer? ¿Tiene cicatrices? ¿Les duelen?

¿Qué ha perdido usted en su vida? (salud, trabajo, familia, amistades, ...)

¿Cuánto tiempo tardaron en cicatrizar esas heridas?

¿Le dejaron alguna huella?

Después de esas pérdidas, ¿le llegaron a su vida situaciones, personas que le causaron bienestar?

Tras esas pérdidas, ¿aprendió usted algo que le ayudara en su vida?

Fase 2:

¿Es difícil para usted soportar el sufrimiento?

¿Qué hace con su sufrimiento?

¿Lo trata de evitar? ¿Cómo lo hace?

¿Se lamenta de lo que ha perdido y se recrea en su sufrimiento?

¿Espera que alguien o algo le salve de su sufrimiento?

¿Cree que no se recuperará nunca?

¿Se aísla?

¿Tiene usted cosas buenas en su vida ahora?

¿Se le olvida que las tiene?

¿Cree usted que no tienen valor?

¿Le parece mal sentirse bien en algún momento?

Fase 3:

¿Siente usted lo que ha perdido?

¿Qué cosas buenas le dio lo que ha perdido?

¿Si alguien le da algún buen consejo procura llevarlo a cabo?

¿Propicia usted cambios para recuperarse?

¿Se relaciona con familia y amistades?

¿Se ocupa?

¿Hace ejercicio?

¿Qué cosas valora en su vida ahora?

¿Cómo las cuida?

Cuando siente algún bienestar, ¿se pregunta por qué eso es así? ¿Procura propiciarlo de nuevo?

Fase 4:

Se responden - en este caso individualmente - a las preguntas formuladas en un Cuestionario de valoración y satisfacción con el tratamiento.

¿En qué grado cree que el tratamiento recibido hoy le ayudará a entender mejor y aceptar lo que le ocurre?

¿En qué grado cree que el tratamiento recibido hoy le ha dado ideas útiles para poder mejorar su situación personal y emocional actual?

¿En qué grado cree que podrá llevar a la práctica dichas ideas útiles?

¿Cuál es un nivel de satisfacción con el tratamiento recibido hoy? 


\section{ANEXO 3}

\section{Guía asistencial con personas que sufren trastornos adaptativos en atención primaria}

\section{Diagnóstico, curso, pronóstico de los trastornos adaptativos}

La CIE-10 define los trastornos de adaptación como estados de malestar subjetivo acompañados de alteraciones emocionales que, por lo general, interfieren con la actividad social y que aparecen en el período de adaptación a un cambio biográfico significativo o a un acontecimiento vital estresante (AVE). La persona siente que no puede afrontar los problemas, planificar el futuro, continuar con la situación presente; sufriendo, en definitiva, un deterioro del cómo se lleva a cabo la rutina diaria.

El cuadro puede incluir síntomas depresivos, ansiosos, preocupación, irritabilidad, agresividad y comportamientos antisociales (no es raro en el caso de adolescentes); y en el caso de niños y niñas conductas regresivas como chuparse el pulgar, utilizar un lenguaje más infantil y enureris nocturna.

El cuadro suele comenzar en el mes posterior a la presentación del AVE y la duración del mismo raramente excede los 6 meses después de que el estresor haya cesado; aunque en el caso de reacciones depresivas puede prolongarse hasta los 2 años. Cuando se supera este período de tiempo es porque existen estresores mantenidos o el cuadro ha devenido a otro de más gravedad.

Si el AVE es relativamente poco importante o no puede ser demostrada una relación temporal entre el cuadro y aquél (menos de 3 meses), el diagnóstico será otro.

Particularmente, los duelos se considerarán trastornos de adaptación si sus manifestaciones son anormales o si son intensas y duran más allá de 6 meses.

En la CIE-10 se distinguen 7 formas específicas de trastornos adaptativos:

Reacción depresiva breve: No excede de 1 mes.

Reacción depresiva prolongada: Hasta 2 años.

Reacción mixta ansiedad-depresión.

Con predominio de alteración de otras emociones: Por ejemplo, preocupación, tensiones e ira.

Con predominio de alteraciones disociales.

Con alteración mixta de emociones y disociales.

Otro trastorno de adaptación con síntomas predominantes especificados.

El pronóstico de los trastornos adaptativos es, en general, bueno y suelen remitir espontáneamente.

\section{Uso de la Guía de autoayuda}

La Guía de Autoayuda que se presenta pretende ser una herramienta de trabajo para la médica y el médico de familia en el proceso de la ayuda psicológica que se le presta al paciente.

En esta Guía probablemente se recojan contenidos que la médica y el médico ya ponen al servicio del paciente en la atención que le prestan. Si es así, el verlos aquí le reafirmarán en su quehacer. Si no lo es, le dará ideas para aumentar su potencial terapeútico psicológico en su consulta.

En todo caso, la Guía no pretende sustituir lo que ya la médica o el médico hace habitualmente durante el tiempo que atiende a la persona en la consulta, sino que, en todo caso, es una prolongación del tratamiento, que se dispensa fuera de ella.

Esta Guía incluye medicamentos psicológicos que la persona debe tomarse en los siguientes meses para encontrase mejor, que debe leer todos los días (como si se tomara un medicamento), despacio, comprensivamente y reflexionando sobre sí y los cambios que tiene que hacer para ponerse en el camino de su recuperación.

Las consultas sucesivas que la persona solicite por su malestar adaptativo conviene que tengan como referencia esta Guía, de manera que, sin desatender aspectos que la médica o el médico consideren relevantes, será el guión de trabajo del paciente para su recuperación. Si se pudiera, convendría que se valore con la 
persona qué dificultades tiene con las indicaciones que se dan en ella, que está poniendo en funcionamiento, qué considera que le está sentando bien, en qué tiene que seguir esmerándose, etc.

\section{Indicación de tratamiento psicológico en grupo de una sola sesión}

La médica o el médico de familia considerará la indicación de tratamiento psicológico grupal en función de la evolución de la persona y del tiempo transcurrido desde que comienza la intervención con la Guía de Autoayuda.

En tal caso, se le informará a la persona que se le indica recibir un tratamiento psicológico en grupo de una sola sesión, que se desarrollará por un facultativo de la USMC-Oriente en el Centro de Salud Puerta Este. También se le dirá que se le incluye en una lista de espera y desde esa Unidad se le llamará para decirle el día, la hora y recordarle el lugar de la sesión.

La médica o el médico de familia no realizará una derivación (solicitud informática de atención en la USMC-Oriente), sino que se comunicará el nombre, el DNI o NHUSA y la fecha de indicación a la/el profesional del Centro de Salud de enlace con la USMC-Oriente. Periódicamente el profesional de esta Unidad que desarrolla la intervención se comunicará con dicha/o profesional para recoger las personas propuestas. Ya desde la USMC-Oriente se les llamará para decirles día y hora de la sesión y recordarle el lugar de realización.

\section{Derivación ordinaria a la usmc-oriente}

La derivación se realizará en alguno de los siguientes supuestos:

- Si al recibir a la persona existen dudas razonables de que esté sufriendo un trastorno de mayor entidad.

- Cuando la persona haya sufrido con anterioridad un trastorno de mayor entidad aunque actualmente pueda estar sufriendo sólo un trastorno adaptativo.

- Cuando, sufriendo un trastorno adaptativo actualmente, también presente otro tipo de trastorno mental simultáneamente.

En estos casos se seguirá el procedimiento habitual cuando se deriva a una persona para que sea valorada en nuestra Unidad. 